\title{
O efeito do polimorfismo TaqIA (rs1800497) no comportamento do tabagismo em um grupo populacional Brasileiro
}

\author{
The effect of the TaqIA polymorphism (rs1800497) on smoking behavior in a Brazilian \\ population group
El efecto del polimorfismo TaqIA (rs1800497) sobre el comportamiento de fumar en un grupo de población Brasileño

Caroline de Lima Mota ${ }^{1 *}$, Simone Mitri1, Cristiane Barata-Silva², Josino Costa Moreira.

\begin{abstract}
RESUMO
Objetivo: Investigar a associação entre um polimorfismo relacionado aos receptores de dopamina, conhecido como TaqIA, e o comportamento tabágico em uma amostra da população Brasileira. Métodos: Trata-se de um estudo transversal, com 449 indivíduos de ambos os sexos, maiores de 18 anos. O histórico do comportamento tabágico foi obtido por meio de um questionário. Amostras de sangue foram genotipadas para TaqIA (rs1800497) por Reação em Cadeia da Polimerase em tempo real. Análises de Regressão logística e linear foram usadas para verificar o efeito dos genótipos no comportamento tabágico. Resultados: $66,4 \%$ dos participantes eram Nunca fumantes, 12,2\% Fumantes atuais e 21,4\% Ex-fumantes. $O$ alelo variante $T$ de TaqIA foi associado ao início do tabagismo mais tardio em comparação a $C C(16,97$ vs. 15,09 ; IC $95 \%: 15,75-18,19 ; p=0,02)$. O alelo $T$ também conferiu maior probabilidade de cessação em mulheres ( $O R=3,17 ; 95 \%$ IC: $1,06-9,45 ; p=0,04)$. Nos homens não foi encontrada diferença significativa. Não foi observada associação entre o polimorfismo e o risco para se tornar fumante, CPD, e FTND. Conclusão: TaqIA rs1800497 foi associado com um efeito protetor para idade de iniciação do tabagismo e maior probabilidade de cessação em mulheres, em uma amostra da população Brasileira.
\end{abstract}

Palavras-chave: Tabagismo, Saúde pública, Receptores de dopamina D2, Abandono do uso de tabaco, Polimorfismo genético.

\section{ABSTRACT}

Objective: To investigate the association between a polymorphism related to dopamine receptors, known as Taq1A, and smoking behavior in a Brazilian population sample. Methods: This is a cross-sectional study, with 449 individuals of both sexes, over 18 years old.The history of smoking behavior was obtained through a questionnaire. Blood samples were genotyped for TaqIA (rs1800497) by real-time Polymerase Chain Reaction (qPCR). Logistic and linear regression analyzes were used to verify the effect of genotypes on smoking behavior. Results: $66.4 \%$ of participants were Never smokers, $12.2 \%$ were Current smokers and $21.4 \%$ were Ex-smokers. The variant $T$ allele of TaqIA was associated with later smoking initiation compared to $C C(16.97$ vs. $15.09 ; 95 \% \mathrm{Cl}: 15.75-18.19 ; \mathrm{p}=$ $0.02)$. The $T$ allele also conferred a higher probability of cessation in women $(\mathrm{OR}=3.17 ; 95 \% \mathrm{Cl}: 1.06-9.45 ; \mathrm{p}=$ 0.04). No significant difference was found in men. No association was observed between the polymorphism and the risk of becoming a smoker, CPD, and FTND. Conclusion: TaqIA rs 1800497 was associated with a protective effect for the age of initiation of smoking and a higher probability of cessation in women, in a Brazilian population sample.

Keywords: Tobacco smoking, Public health, Receptors, Dopamine D2, Tobacco use cessation, Polymorphism genetic.

${ }^{1}$ Centro de Estudos da Saúde do Trabalhador e Ecologia Humana (CESTEH)/ Escola Nacional de Saúde Pública Sergio Arouca (ENSP), Fundação Oswaldo Cruz (FIOCRUZ), Rio de Janeiro - RJ.

*E-mail: carolinelima_bio@yahoo.com.br

2 Instituto Nacional de Controle de Qualidade em Saúde (INCQS), Fundação Oswaldo Cruz (FIOCRUZ), Rio de Janeiro - RJ 


\section{RESUMEN}

Objetivo: Investigar la asociación entre un polimorfismo relacionado con los receptores de dopamina (TaqIA) y el comportamiento de fumar en una muestra de la población Brasileña. Métodos: Este es un estudio transversal, con 449 individuos de ambos sexos, mayores de 18 años. La historia del tabaquismo se obtuvo a través de un cuestionario. Las muestras de sangre se genotiparon para TaqIA (rs1800497) mediante Reacción en Cadena de la Polimerasa (qPCR). Se utilizaron análisis de regresión logística y lineal para verificar el efecto de los genotipos sobre fumar. Resultados: $66.4 \%$ de los participantes eran Nunca fumadores; el $12,2 \%$ Fumadores actuales y el $21,4 \%$ Exfumadores. La alelo $T$ se asoció con el inicio de fumar más tarde en comparación con CC (16.97 vs. 15.09; IC 95\%: 15.75-18.19; $p=0.02)$. El alelo $T$ también confirió mayor probabilidad de cese en las mujeres $(\mathrm{OR}=3.17$; IC 95\%: 1.06-9.45; $p=0.04$ ). No se encontraron diferencias significativas en los hombres. No se observó asociación para el riesgo de convertirse en fumador, CPD, y FTND. Conclusión: TaqIA rs1800497 se asoció con un efecto protector para la edad de inicio del tabaquismo y mayor probabilidad de dejar de fumar en mujeres Brasileñas.

Palabras clave:Tabaquismo, Salud Pública, Receptores de dopamina D2, Cese dal uso de tabaco, Polimorfismo genético.

\section{INTRODUÇÃO}

O tabagismo é um grave problema de Saúde Pública, associado ao desenvolvimento de diversas doenças, incluindo acidente vascular cerebral, distúrbios pulmonares, doenças cardiovasculares e vários tipos de câncer. O fumo de tabaco representa a principal causa de morte evitável no mundo, sendo responsável por 8 milhões de óbitos por ano (WHO, 2020). No Brasil, provoca anualmente mais de 150 mil mortes e configura um alto custo para a economia com despesas médicas, internações e perda de produtividade (PINTO M, et al., 2018).

Apesar do amplo conhecimento dos efeitos adversos para a saúde, a cessação de fumo é um desfecho raro com frequentes recaídas (CDC, 2011; NCCDPHP, 2014; ROCHA EF, et al., 2019; SILVA NBNC, et al., 2019). Estudos mostram que a vulnerabilidade ao tabaco é individual e influenciada por fatores ambientais e genéticos, sendo de aproximadamente $40-75 \%$ para iniciação do fumo, $70-80 \%$ para persistência e mais de $50 \%$ para o sucesso de cessação (LI MD, et al., 2003; MUNAFÒ M, et al., 2004).

Dentre mais de 7000 substâncias químicas aos quais os fumantes de cigarro estão expostos, a nicotina é o principal componente aditivo. Assim como acontece no uso de outras drogas, a nicotina estimula a liberação de dopamina no sistema mesolímbico, que provoca sensação de prazer e recompensa e reforça 0 comportamento da auto-administração (BENOWITZ NL, 2010; MA Y, et al., 2015; NCCDPHP, 2014).

O gene $D R D 2$, que codifica o receptor de dopamina do tipo D2, é um importante componente do sistema de recompensa dopaminérgico, e tem sido associado à susceptibilidade ao tabagismo e outras desordens psiquiátricas (HIRASAWA-FUJITA M, et al., 2017; MA Y, et al., 2015). Os receptores D2 são acoplados à proteína $G$ que inibe a atividade da adenilato ciclase. Nos neurônios pós sinápticos, regulam a propagação do impulso nervoso, enquanto que nos pré-sinápticos têm função de auto-receptores, limitando a liberação de dopamina (DE MEI C, et al., 2009; MA Y, et al., 2015).

Particularmente, um polimorfismo que tem recebido atenção é o TaqlA ( $r$ 1800497C >T), localizado em uma região codificante do gene ANKK1 (ankyrin repeat and kinase domain containing 1), adjacente ao DRD2. O alelo variante $T$ (ou $A 1$ ) do TaqIA está relacionado à menor expressão da proteína D2 (MA Y, et al., 2015; NEVILLE MJ, et al., 2004).

Este polimorfismo tem sido associado a características do comportamento tabágico como a iniciação do tabagismo, o grau de dependência nicotínica, o número de cigarros consumidos por dia (CPD) e o sucesso de cessação. Entretanto, as associações não são consistentemente encontradas (CLAGUE J, et al., 2010; GORDIEV M, et al., 2013; MORTON LM, et al., 2006; RADWAN GN, et al., 2007; TOMAZ PRX, et al., 2015). Na população Brasileira, que tem um perfil genético heterogêneo devido à grande miscigenação étnica (LINS et al., 2009), os trabalhos ainda são escassos (MOURA ACM, 2016; TOMAZ PRX, et al., 2015). 
Neste sentido, o objetivo deste estudo foi verificar a associação entre o polimorfismo TaqIArs1800497 e a susceptibilidade para o fumo, assim como analisar o efeito nas características do comportamento tabágico como o número de cigarros consumidos por dia, idade de iniciação, grau de dependência nicotínica e cessação em uma amostra da população Brasileira.

\section{MÉTODOS}

Este estudo foi do tipo observacional com o delineamento transversal, realizado entre os anos de $2016 \mathrm{e}$ 2019. Em uma amostra de conveniência, a população do estudo foi composta por 449 indivíduos residentes em uma cidade do Rio de Janeiro, Brasil. Os participantes responderam a um questionário de avaliação dos dados sociodemográficos e o histórico do uso de tabaco. Os critérios de elegibilidade foram ser maiores de 18 anos no momento da entrevista e serem capazes de responder ao questionário. Foram excluídos do estudo indivíduos em quem não foi possível coletar amostra biológica.

Por meio do questionário, os participantes foram classificados quanto ao status tabágico em três grupos: Nunca fumantes, Fumantes atuais e Ex-fumantes. Os Nunca fumantes foram definidos como indivíduos que nunca fumaram ou fumaram menos de 100 cigarros durante toda a vida; Fumantes atuais foram indivíduos que fumaram pelo menos 100 cigarros na vida e ainda fumavam regularmente; Ex-fumantes foram aqueles que haviam parado de fumar há pelo menos doze meses. O risco para o fumo foi avaliado pela comparação entre Nunca fumantes e Fumantes atuais + Ex-fumantes. A idade de iniciação do hábito tabágico e a cessação foram avaliadas entre os Fumantes atuais e Ex-fumantes. O grau de dependência, definido pelo Teste dedependência nicotínica de Fagerstrom (FTND), e o número de cigarros consumidos (CPD) foram analisados entre os Fumantes atuais.

Na mesma ocasião da aplicação do questionário, foram coletadas amostras de sangue de cada participante em tubos com EDTA e armazenados a $-80^{\circ} \mathrm{C}$ até o momento da análise. O DNA usado como molde para a amplificação por Reação em Cadeia da Polimerase em tempo real (qPCR) foi extraído a partir de $500 \mu \mathrm{L}$ de sangue total, usando o método baseado na técnica de Salting-Out (SAMBROOK J, 2001). Após a extração, o DNA foi quantificado utilizando o espectofotômetro NanoDrop 2000 (Thermo Fisher ScientificTM) e o polimorfismo TaqIA foi genotipado pelo sistema de discriminação alélica TaqMan (ensaio: rs 1800497; C_7486676_10), em equipamento de PCR em Tempo Real (Reação em Cadeia da Polimerase) (7500 RealTime PCR System, Applied Biosystems) de acordo com os protocolos do fabricante. Para verificar a eficiência da genotipagem, $10 \%$ das amostras foram aleatoriamente selecionadas e refeitas. Não houve discrepância dos resultados.

A análise dos dados foi realizada utilizando-se o programa estatístico SPSS 20.0 (Statistical Package for Social Sciences Inc., Chicago, IL, USA). Para verificar a normalidade das variáveis foi utilizado o teste Kolmogorov-Smirnov. O equilíbrio de Hardy-Weinberg (HWE) foi avaliado pelo teste Qui-quadrado (X2). As variáveis contínuas foram apresentadas como média e desvio-padrão (DP), enquanto as categóricas foram apresentadas por frequências. O Teste de Kruskal-Wallis e o Teste $\mathrm{X} 2$ foram usados para avaliar diferenças nas médias e frequências.

Os genótipos do TaqIA rs1800497 foram classificados baseado em estudos prévios (GORDIEV M, et al., 2013; SWAN GE, et al., 2005). Primeiramente, $C C$ foi utilizadocomo referência para comparação com $C T$ e $T T$ ( $C C$ versus $C T$; $C C$ versus $T T$ ). Adicionalmente, devido à baixa frequência de $T T$, os genótipos foram agrupados pela presença do alelo variante $T$ (CT ou $T T$ versus $C C$ ).

O teste de Regressão logística foi utilizado para avaliar a possível associação entre TaqIA e o risco para o fumo, considerando o status tabágico como variável dependente e ajustado pela idade, sexo, escolaridade (anos de estudo), consumo de álcool (sim/não) e cor da pele (auto declarada). A análise da chance de cessação espontânea do tabagismo (no grupo estudado não houve interferência medicamentosa) também foi realizada por Regressão logística, ajustada pelo sexo, consumo de álcool, cor da pele e idade de iniciação do fumo. As associações entre o polimorfismo e a idade de início, o número de cigarros consumidos por dia (CPD) e o grau de dependência nicotínica segundo Fagerstrom (FTND) foram estimadas pela comparação de médias, com ajuste para possíveis fatores de confundimento, utilizando o modelo de Regressão linear. $O$ nível de significância estatística considerado para todas as análises foi de $p \leq 0,05$. 
O estudo foi aprovado pelo Comitê de ética em pesquisa da Escola Nacional de Saúde Pública Sérgio Arouca/ Fundação Oswaldo Cruz (ENSP, FIOCRUZ), com o número CAAE 40514415.0.0000.5240 e parecer número 2.090.482. Os participantes foram informados e esclarecidos sobre os objetivos e procedimentos da pesquisa e a participação foi voluntária atendendo à Resolução 466/2012 do Conselho Nacional de Saúde. Todos os participantes assinaram o termo de consentimento livre e esclarecido (TCLE).

\section{RESULTADOS}

Dos 449 indivíduos que participaram do estudo, 66,4\% eram Nunca fumantes ( $n=298)$ e 12,2\% eram Fumantes atuais $(n=55)$ e $21,4 \%$ eram Ex-fumantes $(n=96)$. A Tabela 1 apresenta as características sociodemográficas da população do estudo de acordo com o status tabágico. A idade variou entre 18 a 87 anos e $68,2 \%$ eram mulheres $(n=306)$. A maior parte da população se declarou não branca $(74,0 \%)$, que incluiu pretos, pardos, e descendentes de indígenas e Asiáticos. Entre os Fumantes atuais, 70,9\% reportaram consumir bebida alcoólica, enquanto entre os Nunca fumantes e Ex-fumantes, a frequência do uso de álcool foi menor, $40,9 \%$ e 42,7\%, respectivamente. A renda familiar da maioria dos participantes foi menor do que 3 salários mínimos, que no início da pesquisa era $R \$ 937,00$ reais. Foram encontradas diferenças significativas para gênero, idade, escolaridade e consumo de álcool $(p<0,05)$.

Tabela 1 - Características sociodemográficas da população do estudo, n=449, 2016-2019.

\begin{tabular}{|c|c|c|c|c|c|}
\hline Variável & $\begin{array}{l}\text { Total } \\
\text { n (\%) }\end{array}$ & $\begin{array}{c}\text { Nunca } \\
\text { fumantes } \\
\mathrm{n}(\%)\end{array}$ & $\begin{array}{c}\text { Fumantes } \\
\text { atuais } \\
\mathbf{n}(\%)\end{array}$ & $\begin{array}{c}\text { Ex- } \\
\text { fumantes } \\
n(\%)\end{array}$ & $\mathbf{p}$ \\
\hline \multicolumn{6}{|l|}{ Sexo } \\
\hline Feminino & $306(68,2 \%)$ & $216(72,5)$ & $31(56,4)$ & $59(61,5)$ & \multirow{2}{*}{0,02} \\
\hline masculino & $143(31,8 \%)$ & $82(27,5)$ & $24(43,6)$ & $37(38,5)$ & \\
\hline Idade (média; DP) & $51,96(15,12)$ & $50,37(15,71)$ & $51,02(12,75)$ & $57,45(13,21)$ & $<0,01$ \\
\hline Escolaridade (média; DP) & $8,59(3,95)$ & $8,95(3,91)$ & $8,35(3,68)$ & $7,64(4,08)$ & 0,01 \\
\hline \multicolumn{6}{|l|}{ Cor da pele ${ }^{1}$} \\
\hline Branco & $116(26,0 \%)$ & $85(28,7)$ & $9(16,4)$ & $22(23,2)$ & \multirow{2}{*}{0,12} \\
\hline Não Branco² & $330(74,0 \%)$ & $211(71,3)$ & $46(83,6)$ & $73(76,8)$ & \\
\hline \multicolumn{6}{|l|}{ Uso de álcool } \\
\hline Sim & $202(45,0 \%)$ & $122(40,9)$ & $39(70,9)$ & $41(42,7)$ & \multirow{2}{*}{$<0,01$} \\
\hline Não & $247(55,0 \%)$ & $176(59,1)$ & $16(29,1)$ & $55(57,3)$ & \\
\hline \multicolumn{6}{|l|}{ Renda $^{2}{ }^{3}$} \\
\hline Menos de 3 & $258(69,5)$ & $170(68,5)$ & $31(81,6)$ & $57(67,1)$ & \multirow{4}{*}{0,68} \\
\hline De 3 a menos de 5 & $95(25,6)$ & $65(26,2)$ & $7(18,4)$ & $23(27,1)$ & \\
\hline De 5 a menos de 10 & $15(4,0)$ & $11(4,4)$ & $0(0,0)$ & $4(4,7)$ & \\
\hline 10 ou mais & $3(0,8)$ & $2(0,8)$ & $0(0,0)$ & $1(1,2)$ & \\
\hline Total & 449 & 298 & 55 & 96 & \\
\hline
\end{tabular}

Legenda: DP, desvio padrão; $p$ valor obtido pelos Teste Qui-quadrado (x2) e Kruskal-Wallis; ${ }^{1} \mathrm{O}$ valor de $\mathrm{n}$ variou devido ao dado faltante (cor da pele $n=3$; renda $n=78$ ); Pretos, pardos, descendentes de indígenas e Asiáticos;

Fonte: Mota CL, et al., 2020.

A distribuição genotípica de TaqIA estava de acordo com o equilíbrio de Hardy-Weinberg (HWE). A frequência encontrada foi de 50,6\% $(n=227)$ para o CC, 39,6\% $(n=178)$ para CT e 9,8\% $(n=44)$ para TT. A frequência observada para o alelo variante $T$ foi de $29,6 \%$.

O resultado da associação entre o polimorfismo TaqIArs1800497 e o risco de ser tabagista foi estimado pela análise de Regressão logística. Foi observada uma pequena diferença nas frequências genotípicas entre 
os grupos. Os fumantes atuais e Ex-fumantes apresentaram uma menor prevalência do alelo variante $T$ $(46,4 \%)$, do que os Nunca fumantes $(51 \%)$, entretanto a diferença encontrada não foi estatisticamente significativa ( $C$ C versus CT/TT: OR= 0,$79 ; 95 \%$ IC: $0,52-1,20 ; \mathrm{p}=0,26$ ) (Tabela 2). Também não foi encontrada associação significativa quando se estratificou a amostra pelo sexo (dados não mostrados).

Tabela 2 - Associação entre TaqIA rs1800497 e o risco para o tabagismo, n=449, 2016-2019.

\begin{tabular}{ccccc}
\hline Genótipo & $\begin{array}{c}\text { Nunca fumantes } \\
(\mathbf{n = 2 9 8 )}\end{array}$ & \begin{tabular}{c} 
Fumantes atuais + Ex-fumantes \\
$(\mathbf{n = 1 5 1 )}$ \\
\cline { 2 - 3 } $\mathbf{n}(\%)$
\end{tabular} & OR (IC 95\%) & p \\
\cline { 2 - 3 }$C C$ & $\mathbf{n}(\%)$ & $81(53,6)$ & $1^{\text {a }}$ & \\
$C T$ & $146(49,0)$ & $55(36,4)$ & $0,80(0,51-1,24)$ & 0,32 \\
$T T$ & $123(41,3)$ & $15(9,9)$ & $0,74(0,35-1,54)$ & 0,42 \\
$C T / T T$ & $29(9,7)$ & $70(46,4)$ & $0,79(0,52-1,20)$ & 0,26 \\
\hline
\end{tabular}

Legenda: OR, odds ratio (razão de chance) ajustada por idade, sexo, escolaridade, consumo de álcool e cor da pele; IC, Intervalo de confiança. p valor obtido por Regressão logística.a, Grupo de referência.

Fonte: Mota CL, et al., 2020.

A Tabela 3 apresenta a análise de Regressão linear para a idade de iniciação, grau de dependência segundo Fagerstrom (FTND) e número de cigarros consumidos por dia (CPD) de acordo com TaqIA rs 1800497. O alelo variante $T$ foi associado à idade de iniciação do fumo mais tardia, em comparação ao genótipo CC (16,97 vs. 15,09; IC 95\%: 15,75-18,19; $\mathrm{p}=0,02)$. Também foi observado que indivíduos heterozigotos $C T$ começaram a fumar em média 1,76 anos mais tarde do que CC (IC 95\%: 15,42-18,28; $p=$ $0,04)$. A média de iniciação foi ainda mais alta para portadores do genótipo homozigoto variante $T T$, de 2,31 anos (IC 95\%: 14,84-19,96; $p=0,02$ ). Não foi encontrada associação significativa quando se estratificou a amostra pelo sexo (dados não mostrados). Da mesma forma, FTND e CPD também não foram associados ao polimorfismo ( $p>0.05)$ (Tabela 3$)$.

Tabela 3 - Associação entre TaqIA rs1800497 e características do comportamento tabágico, n=146 para Idade de início, $\mathrm{n}=51$ para CPD e FTND, 2016-2019.

\begin{tabular}{|c|c|c|c|c|}
\hline \multirow[b]{2}{*}{ Genótipo } & \multicolumn{4}{|c|}{ Idade de início ( $\mathrm{n}=146)$} \\
\hline & $\mathbf{n}$ & Média & IC 95\% & $\mathbf{p}$ \\
\hline$C C$ & 78 & 15,09 & $14,21-15,97$ & $1^{\mathrm{a}}$ \\
\hline$C T$ & 53 & 16,85 & $15,42-18,28$ & 0,04 \\
\hline$T T$ & 15 & 17,40 & $14,84-19,96$ & 0,02 \\
\hline \multirow[t]{3}{*}{$C T / T T$} & 68 & 16,97 & $15,75-18,19$ & 0,02 \\
\hline & \multicolumn{4}{|c|}{ Grau de dependência nicotínica segundo Fagerstrom(FTND) ( $n=51)$} \\
\hline & $\mathbf{n}$ & Média & IC 95\% & $\mathbf{p}$ \\
\hline$C C$ & 30 & 4,47 & $3,52-5,41$ & $1^{a}$ \\
\hline$C T$ & 18 & 3,83 & $2,64-5,03$ & 0,35 \\
\hline$T T$ & 3 & 4,33 & $2,84-11,50$ & 0,92 \\
\hline \multirow{3}{*}{ CT/TT } & 21 & 3,90 & $2,81-5,00$ & \\
\hline & & \multicolumn{3}{|c|}{ Número de cigarros consumidos por dia (CPD) $(n=51)$} \\
\hline & $\mathbf{n}$ & Média & IC 95\% & $\mathbf{p}$ \\
\hline$C C$ & 30 & 15,67 & $12,94-18,39$ & $1^{a}$ \\
\hline$C T$ & 18 & 13,50 & $8,69-18,31$ & 0,37 \\
\hline$T T$ & 3 & 17,00 & $13,53-47,53$ & 0,89 \\
\hline$C T / T T$ & 21 & 14,00 & $9,54-18,46$ & 0,38 \\
\hline
\end{tabular}

Legenda: IC, Intervalo de confiança da média; $p$ valor obtido por Regressão linear.Idade de início: ajustado por sexo e consumo de álcool; FTND (grau de dependência segundo Fagerstrom) e CPD (cigarros por dia):ajustado por sexo, consumo de álcool e idade.a, Grupo de referência.

Fonte: Mota CL, et al., 2020.

A análise da associação entre a cessação do tabagismo e TaqlA verificada por meio de Regressão logística mostrou uma probabilidade de cessação 3,17 vezes maior em mulheres portadoras do alelo variante 
$T$ em comparação com $C C$ (CC versus $C T / T T$ : OR=3,17; 95\% IC: 1,06-9,45; $p=0,04)$. Na população geral e no grupo masculino não foram encontradas associações significativas $(p>0,05)$. Devido à baixa frequência do genótipo variante após a estratificação pelo sexo, a avaliação da cessação foi realizada utilizando o modelo de agrupamento dos genótipos já definido previamente, CC versus CT/TT (Tabela 4).

Tabela 4 - Associação entre TaqIA rs1800497 e a cessação do tabagismo, n=151, 2016-2019.

\begin{tabular}{|c|c|c|c|c|}
\hline \multicolumn{5}{|c|}{ População total $(\mathrm{n}=151)$} \\
\hline \multirow{2}{*}{ Genótipo } & Fumantes atuais( $n=55)$ & Ex-fumantes $(n=96)$ & & \\
\hline & n (\%) & n (\%) & OR(IC 95\%) & $\mathbf{p}$ \\
\hline$C C$ & $32(58,2)$ & $49(51,0)$ & $1^{a}$ & \multirow{2}{*}{0,07} \\
\hline$C T / T T$ & $23(41,8)$ & $47(49,0)$ & $2,04(0,94-4,45)$ & \\
\hline \multicolumn{5}{|c|}{ Mulheres $(n=90)$} \\
\hline \multirow{2}{*}{ Genótipo } & Fumantes atuais $(n=31)$ & Ex-fumantes(n=59) & & \\
\hline & n (\%) & n (\%) & OR(IC 95\%) & p \\
\hline$C C$ & $18(58,1)$ & $28(47,5)$ & $1^{a}$ & \multirow[b]{2}{*}{0,04} \\
\hline$C T / T T$ & $13(41,9)$ & $31(52,5)$ & $3,17(1,06-9,45)$ & \\
\hline & \multicolumn{2}{|c|}{ Homens $(n=61)$} & & \\
\hline \multirow[t]{2}{*}{ Genótipo } & Fumantes atuais $(n=24)$ & Ex-fumantes(n=37) & & \\
\hline & n (\%) & n (\%) & OR(IC 95\%) & $\mathbf{p}$ \\
\hline$C C$ & $14(58,3)$ & $21(56,8)$ & $1^{a}$ & \\
\hline$C T / T T$ & $10(41,7)$ & $16(43,2)$ & $\begin{array}{c}1,39 \\
(0,43-4,54)\end{array}$ & 0,58 \\
\hline
\end{tabular}

Legenda: OR, odds ratio (razão de chance) ajustada por sexo, consumo de álcool, cor da pele e idade de iniciação do tabagismo; IC, Intervalo de confiança.p valor obtido por Regressão logística.a, Grupo de referência. Fonte: Mota CL, et al., 2020.

\section{DISCUSSÃO}

Neste estudo foi realizada a análise da associação entre o polimorfismo TaqIA rs1800497 e o comportamento tabágico em uma amostra da população Brasileira. Este polimorfismo tem sido associado à desordens psiquiátricas e ao mecanismo de recompensa relacionado às drogas de abuso, incluindo o álcool e o tabaco (HUANG W, et al., 2009; MA Y, et al., 2015).

Algumas hipóteses são sugeridas para a relação entre o polimorfismo TaqlA rs1800497 e a susceptibilidade ao tabaco. Pesquisas reportam que portadores do alelo variante $T$ do TaqIA possuem uma menor expressão da proteína D2 (MA Y, et al., 2015; NEVILLE MJ, et al., 2004). Devido ao papel de autoreceptor desempenhado pela proteína, que limita a taxa de síntese do neurotransmissor, a menor densidade dos receptores D2 estaria relacionada a um maior nível de dopamina sináptica.

Desta forma, indivíduos poradores de alelo $T$ do TaqIA teriam menor necessidade da repetição da estimulação dopaminérgica do que indivíduos que não apresentam a variação polimórfica, sendo portanto, menos susceptíveis à adição a nicotina. Entretanto, a função do TaqIA ainda não é completamente elucidada e mais estudos são necessários para compreenção dos mecanismos moleculares (BIDWELL LC, et al., 2015; MOURA ACM, 2016).

A literatura mostra que a prevalência do alelo variante $T$ diferentre grupos étnicos. Por exemplo, a frequência é significativamente mais alta em Americanos (53\% a $75 \%$ ) do que em Asiáticos (11\% a 58\%) (OHMOTO M, et al., 2013; RADWAN GN, et al., 2007). Sabe-se que o Brasil é um país com população altamente miscigenada (LINS TC, et al., 2009). No presente estudo, a maior parte dos participantes se declararam pretos, pardos, descendentes de indígenas e Asiáticos (74\%). A frequência do alelo variante $T$ encontrada $(29,6 \%)$ foi semelhante a observada em pesquisas prévias que também envolviam população Brasileira (MOURA ACM, 2016; TOMAZ PRX, et al., 2015).

A relação entre o polimorfismo TaqIA rs1800497 e o tabagismo é controversa. Alguns estudos com população Caucasiana sugerem que o alelo $T$ está associado ao aumento do risco de se tornar fumante 
(COMINGS DE, et al., 1997; MORTON LM, et al., 2006; NOBLE EP, et al., 1994; OHMOTO M, et al., 2013), enquanto pesquisas com Japoneses, Poloneses e Russos mostraram um maior risco para o genótipo CC (GORDIEV M, et al., 2013; HAMAJIMA N, et al., 2002; OHMOTO M, et al., 2013; SIEMINSKA A, et al., 2009; YOSHIDA K, et al., 2001). No presente estudo, não foi encontrada influência significativa do polimorfismo TaqIA para o risco de ser fumante. Entretanto, as frequências genotípicas observadas corroboram com um trabalho anterior, desenvolvido também com uma população Brasileira, que mostrou uma maior frequência do genótipo selvagem $C C$ em Fumantes do que em Não fumantes, em comparação ao alelo variante $T$ (MOURA ACM, 2016).

A influência de TaqIA no comportamento tabágico foi avaliado pela idade de iniciação do hábito, número de cigarros consumidos por dia (CPD) e grau de dependência nicotínica (FTND). Para CPD e FTND não foram encontradas diferenças significativas na distribuição dos genótipos de TaqIA. Estes achados concordam com alguns estudos (BIDWELL LC, et al., 2015; TOMAZ PRX, et al., 2015; RADWAN GN, et al., 2007) mas conflitam com outros trabalhos que sugerem a influência de TaqIA rs 180049 no comportamento do tabagismo (CLAGUE J, et al., 2010; DE RUYCK K, et al., 2010; HUANG W, et al., 2009).

Esta incosistência nos resultados pode ser atribuída a alguns fatores, como por exemplo os métodos de classificação do fumo, a estratificação da amostra estudada, e as diferenças étnicas entre as populações (OHMOTO M, et al., 2013; RADWAN DN, et al., 2007). Além desses fatores, no presente estudo, o número de cigarros consumidos por dia e grau de dependência nicotínica foram analisados apenas no subgrupo de fumantes atuais, o que reduziu o tamanho amostral e pode ter enfraquecido o poder estatístico das análises.

Em relação a idade de iniciação do tabagismo, foi observado entre os Fumantes atuais e Ex-fumantes que portadores do alelo variante $T$ começaram a fumar aproximadamente 2 anos mais tarde do que indivíduos CC. Semelhantemente, outro estudo com amostra Brasileira observou que o alelo variante de TaqIA rs 1800497 tem um efeito protetor na idade de iniciação (MOURA ACM, 2016). Já em Caucasianos, o alelo $T$ parece ser fator de risco para iniciação mais precoce (CLAGUE J, et al., 2010; OHMOTO M, et al., 2013; SPITZ MR, et al., 1998). Os resultados aqui discutidos corroboram com a hipótese já sugerida por outros autores sobre a influência das diferenças étnicas na relação entre TaqIA e o comportamento do tabagismo (OHMOTO M, et al., 2013).

O estudo também mostrou associação entre o alelo variante $T$ de TaqIA e maior probabilidade de cessação em mulheres. Este resultado é consistente com pesquisas prévias que observaram que, em comparação com o genótipo selvagem $C C$, indivíduos portadores de pelo menos um alelo variante $T$ tinham maior chance de parar de fumar (BREITLING LP, et al., 2010; GORDIEV M, et al., 2013; JOHNSTONE EC, et al., 2004; MORTON LM, et al., 2006; SWAN GE, et al., 2005), embora existam achados discordantes(DAVID SP, et al., 2007; LERMAN C, et al., 2003; TOMAZ PRX, et al., 2015).

Quando se analisou a população total e o grupo masculino, não foram encontradas diferenças significativas. Estes resultados sugerem o gênero como um provável fator relevante para a cessação. Várias pesquisas evidenciam que a relação genética-tabagismo difere entre homens e mulheres (OHMOTO M, et al., 2013; PETERSEN N e LONDON ED, 2018; SWAN, et al., 2005). Yudkin P, et al. (2004) confirmam estas evidências, ao observarem uma considerável eficácia no tratamento para cessação com reposição de nicotina em mulheres portadores do alelo variante $T$ de TaqIA. Nos homens, nenhuma relação significante entre os genótipos e o tratamento foi observada (YUDKIN P, et al., 2004).

O efeito do TaqIA rs1800497 na cessação do hábito tabágico também parece ser alterado por interações gene-gene com outras variações polimórficas (JOHNSTONE EC, et al., 2004; LERMAN C, et al., 2003; TON TGN, et al., 2007). Este tipo de investigação foi realizada por Ton TGN, et al. (2007) em uma população com 593 mulheres fumantes de origem caucasiana. Neste estudo, não foi observada associação entre a probabilidade de cessação e TaqIA quando o polimorfismo foi analizado individualmente.

Um efeito significativo para TaqIA foi encontrado apenas quando analizado concomitantemente com um polimorfismo conhecido como SLC6A3 VNTR, presente no gene que codifica a proteína transportadora de dopamina, relacionada a recaptação do neurotransmissor da fenda sináptica. Mulheres portadoras do alelo 
variante $T$ de $T a q I A$ e do genótipo de 9 repetições do polimorfismo SLC6A3 tinham maior probabilidade de parar de fumar em comparação com as outras possibilidades genotípicas.

A principal limitação deste estudo foi o tamanho amostral relativamente pequeno e a amostragem por conveniência. Este tipo de abordagem foi adotada por ser a única possível devido ao acesso restrito à comunidade. O trabalho contribui significativamente para o conhecimento da relação entre variações genéticas no sistema de recompensa dopaminérgico e o comportamento do tabagismo, e se assemelha com resultados de pesquisas com maiores populações. Este estudo é de particular interesse pois envolve a população Brasileira que é altamente miscigenada e pouco estudada. Estudos futuros envolvendo interação gênica e a combinação entre genótipos também são importantes para ajudar no entendimento das bases moleculares da neurobiologia do fumo e contribuir para investigação mais aprofundada da medicina personalizada para a cessação.

\section{CONCLUSÃO}

Em conclusão, o alelo variante $T$ do polimorfismo TaqIA (rs1800497) foi associado com idade de iniciação do fumo mais tardia. Além disso, a variação polimórfica conferiu maior probabilidade de cessação do tabagismo nas mulheres, mostrando que o gênero é um fator de influência para o efeito de TaqIA no sucesso do abandono do hábito. Estes achados contribuem com a literatura sugerindo que o polimorfismo TaqIArs 1800497 confere proteção contra o tabagismo na população Brasileira, e pode auxiliar no avanço dos tratamentos para cessação.

\section{FINANCIAMENTO}

Este estudo foi financiado pela escola de Saúde Pública Sérgio Arouca/ Fundação Oswaldo Cruz (ENSP/FIOCRUZ) (ENSP-018-FIO-17) e pela Fundação de Amparo à Pesquisa do Estado do Rio de Janeiro (FAPERJ) (E-26/200.618/2018), Brasil.

\section{REFERÊNCIAS}

1. BENOWITZ NL. Nicotine addiction. The New England Journal of Medicine, 2010; 362(24): 2295-2303.

2. BIDWELL LC, et al. NCAM1-TTC12-ANKK1-DRD2 variants and smoking motives as intermediate phenotypes for nicotine dependence. Psychopharmacology, 2015; 232(7):1177-86

3. BREITLING LP, et al. Prospective association of dopamine-related polymorphisms with smoking cessation in general care. Pharmacogenomics, 2010; 11(4): 527-536.

4. CENTERS FOR DISEASE CONTROL AND PREVENTION (CDC). Quitting smoking among adults--United States, 2001-2010. MMWR. Morbidity and mortality weekly report, 2011; 60(44): 1513-1519.

5. CLAGUE J, et al. The D2 Dopamine Receptor Gene and Nicotine Dependence Among Bladder Cancer Patients and Controls. Behavior Genetics, 2010; 40(1): 49-58.

6. COMINGS DE, et al. Studies of the potential role of the dopamine D1 receptor gene in addictive behaviors. Molecular Psychiatry, 1997; 2(1):44-56.

7. DAVID SP, et al. Pharmacogenetic clinical trial of sustained-release bupropion for smoking cessation. Nicotine \& Tobacco Research: Official Journal of the Society for Research on Nicotine and Tobacco, 2007; 9(8): 821-833.

8. DE MEI C, et al. Getting specialized: presynaptic and postsynaptic dopamine D2 receptors. Current Opinion in Pharmacology, 2009; 9(1): 53-58

9. DE RUYCK K, et al. Genetic variation in three candidate genes and nicotine dependence, with drawal and smoking cessation in hospitalized patients. Pharmacogenomics, 2010;11(8):1053-63

10. GORDIEV M, Genetic analysis of polymorphisms in dopamine receptor and transporter genes for association with smoking among cancer patients. European Addiction Research, 2013;19(2):105-111.

11. HAMAJIMA N, et al. Association between smoking habits and dopamine receptor D2 taql A A2 allele in Japanese males: a confirmatory study. Journal of Epidemiology, 2002;12(4):297-304.

12. HIRASAWA-FUJITA M, et al. Genetic Variation of the Mu Opioid Receptor (OPRM1) and Dopamine D2 Receptor (DRD2) is Related to Smoking Differences in Patients with Schizophrenia but not Bipolar Disorder. Clinical Schizophrenia \& Related Psychoses, 2017; 11(1): 39-48

13. HUANG W, et al. Significant association of ANKK1 and detection of a functional polymorphism with nicotine dependence in an African-American sample. Neuropsychopharmacology, 2009; 34(2):319-30

14. JOHNSTONE EC, et al. Genetic variation in dopaminergic pathways and short-term effectiveness of the nicotine patch. Pharmacogenetics, 2004; 14(2): 83-90. 
15. LERMAN C, et al. Effects of dopamine transporter and receptor polymorphisms on smoking cessation in a bupropion clinical trial. Health Psychology: Official Journal of the Division of Health Psychology, American Psychological Association, 2003;22(5):541-548.

16. LI MD, et al. A meta-analysis of estimated genetic and environmental effects on smoking behavior in male and female adult twins. Addiction (Abingdon, England), 2003; 98(1): 23-31.

17. LINS TC, et al. Genetic composition of Brazilian population samples based on a set of twenty-eight ancestry informative SNPs. American Journal of Human Biology, 2010; 22(2): 187-192.

18. MA Y, et al. The significant association of Taq1A genotypes in DRD2/ANKK1 with smoking cessation in a large-scale meta-analysis of Caucasian populations. Translational Psychiatry, 2015; 5(12): e686-e686.

19. MORTON LM, et al. DRD2 genetic variation in relation to smoking and obesity in the Prostate, Lung, Colorectal, and Ovarian Cancer Screening Trial: Pharmacogenetics and Genomics, 2006;16(12): 901-910.

20. MOURA ACM, 2016. Prevalência do polimorfismo Taq1a no gene do receptor de dopamina do tipo D2 e seus possíveis efeitos em uma população de tabagistas do nordeste do Brasil, PI. Dissertação (mestrado em Biotecnologia) Universidade Federal do Piauí, Parnaíba, 2016; 86 p.

21. MUNAFÒ M, et al. The genetic basis for smoking behavior: a systematic review and meta-analysis. Nicotine \& Tobacco Research: Official Journal of the Society for Research on Nicotine and Tobacco, 2004;6(4):583-597.

22. NATIONAL CENTER FOR CHRONIC DISEASE PREVENTION AND HEALTH PROMOTION (US) OFFICE ON SMOKING AND HEALTH (NCCDPHP). The Health Consequences of Smoking -50 Years of Progress: A Report of the Surgeon General. Atlanta (GA): Centers for Disease Control and Prevention (US), 2014; 36P.

23. NEVILLE MJ, et al. Identification and characterization of ANKK1: A novel kinase gene closely linked to DRD2 on chromosome band 11q23.1. Human Mutation, 2004;23(6):540-545...

24. NOBLE EP, et al. D2 dopamine receptor gene and cigarette smoking: a reward gene? Medical Hypotheses, 994; 42(4): 257-260.

25. OHMOTO M, et al. Association Between Dopamine Receptor 2 TaqlA Polymorphisms and Smoking Behavior With an Influence of Ethnicity: A Systematic Review and Meta-Analysis Update. Nicotine \& Tobacco Research, 2013; 15(3): 633-642.

26. PETERSEN N, LONDON ED. Addiction and dopamine: sex differences and insights from studies of smoking. Current Opinion in Behavioral Sciences, 2018; 23:150-9.

27. PINTO M, et al. Carga de doença atribuível ao uso do tabaco no Brasil e potencial impacto do aumento de preços por meio de impostos. Documento técnico IECS N²1. Instituto de Efectividad Clínica y Sanitaria, Buenos Aires, Argentina. Maio de 2017. Disponível em: <https://www.inca.gov.br/publicacoes/livros/carga-de-doenca-atribuivel-ao-uso-dotabaco-no-brasil-e-potencial-impacto-do>. Acesso em: 22 abr. 2020.

28. RADWAN GN, et al. DRD2/ANKK1 Taql polymorphism and smoking behavior of Egyptian male cigarette smokers. Nicotine \& Tobacco Research: Official Journal of the Society for Research on Nicotine and Tobacco, 2007;9(12):13251329

29. ROCHA EF, et al. O cigarro, o narguilé e a doença periodontal. Revista Eletrônica Acervo Saúde, 2019; (28): e784.

30. SAMBROOK J. Molecular cloning: a laboratory manual. 3rd ed. Cold Spring Harbor, N.Y: Cold Spring Harbor Laboratory Press; 2001. 3 p.

31. SIEMINSKA A, et al. Influences of polymorphic variants of DRD2 and SLC6A3 genes, and their combinations on smoking in Polish population. BMC medical genetics, 2009; 10:92.

32. SILVA NBN, et al. Tabagismo como fator de risco para o desenvolvimento de câncer de pulmão. Revista Eletrônica Acervo Saúde, 2019; (19): e313.

33. SPITZ MR, et al. Case-control study of the D2 dopamine receptor gene and smoking status in lung cancer patients. Journal of the National Cancer Institute, 1998; 90(5): 358-363.

34. SWAN GE, et al. Dopamine receptor DRD2 genotype and smoking cessation outcome following treatment with bupropion SR. The Pharmacogenomics Journal, 2005; 5(1): 21-9.

35. TOMAZ PRX, et al. CYP2B6 rs2279343 polymorphism is associated with smoking cessation success in bupropion therapy. European Journal of Clinical Pharmacology, 2015; 71(9): 1067-73.

36. TON TG, et al. Genetic polymorphisms in dopamine-related genes and smoking cessation in women: a prospective cohort study. Behavioral and Brain Function, 2007; 3(1): 22.

37. WORLD HEALTH ORGANIZATION. WORLD HEALTH ORGANIZATION (WHO). Who Report On The Global Tobacco Epidemic 2019: offer help to quit tobacco use. Geneva: World Health Organization, 2020; 119p.

38. YOSHIDA K, et al. Association between the dopamine D2 receptor A2/A2 genotype and smoking behavior in the Japanese. Cancer Epidemiology, Biomarkers \& Prevention: A Publication of the American Association for Cancer Research, Cosponsored by the American Society of Preventive Oncology, 2001; 10(4): 403-405.

39. YUDKIN P, et al. Effectiveness of nicotine patches in relation to genotype in women versus men: randomised controlled trial. BMJ (Clinical research ed.), 2004; 328(7446):989-90. 